\title{
Multiple current sheets in a double auroral oval observed from the MAGION-2 and MAGION-3 satellites
}

\author{
M. Echim ${ }^{1}$, M. Ciobanu ${ }^{1}$, O. Balan ${ }^{1}$, A. Blagau ${ }^{1}$, O. Marghitu ${ }^{1}$, E. Georgescu ${ }^{1}$, Y. I. Galperin ${ }^{2}$, N. V. Jorgio ${ }^{2}$, \\ T. M. Muliarchik ${ }^{2}$, A. L. Kotikov ${ }^{3}$, E. M. Shishkina ${ }^{3}$, O. A. Troshichev ${ }^{3}$ \\ ${ }^{1}$ Institute of Gravitation and Space Sciences, P.O. Box MG-06, R-76900, Magurele-Bucharest, Romania \\ ${ }^{2}$ Space Research Institute of RAS, Moscow, 117810 \\ ${ }^{3}$ Institute of Arctic and Antarctic, Roskomgydromet, St. Petersburg, 199226
}

Received: 12 April 1996 / Revised: 6 September 1996 / Accepted: 26 September 1996

\begin{abstract}
A case is described of multiple current sheets crossed by the MAGION-2 satellite in the near-midnight quieting auroral oval. The data were obtained by the magnetometer experiment onboard. Results show during a quieting period after a preceding substorm, or during an early growth phase of the next substorm, two double-sheet current bands, POLB and EQUB, located at respectively the polar and equatorial borders of the auroral oval separated by about $500 \mathrm{~km}$ in latitude. This is consistent with the double-oval structure during recovery introduced by Elphinstone et al. (1995). Within the POLB, the magnetic field data show simultaneous existence of several narrow parallel bipolar current sheets within the upward current branch (at 69.5-70.3 invariant latitude) with an adjacent downward current branch at its polar side at $\left(70.5-71.3^{\circ}\right)$. The EQUB was similarly stratified and located at $61.2-63.5^{\circ}$ invariant latitude. The narrow current sheets were separated on average by about $35 \mathrm{~km}$ and $15 \mathrm{~km}$, respectively, within the POLB and EQUB. A similar case of double-oval current bands with small-scale structuring of their upward current branches during a quieting period is found in the data from the MAGION-3 satellite. These observations contribute to the double-oval structure of the late recovery phase, and add a small-scale structuring of the upward currents producing the auroral arcs in the double- oval pattern, at least for the cases presented here. Other observations of multiple auroral current sheets and theories of auroral arc multiplicity are briefly discussed. It is suggested that multiple X-lines in the distant tail, and/or leakage of energetic particles and FA currents from a series of plasmoids formed during preceding magnetic activity, could be one cause of highly stratified upward FA currents at the polar edge of the quieting double auroral oval.
\end{abstract}

Correspondence to: $\mathrm{M}$. Echim

\section{Introduction}

Auroral observers often noted that visible arcs in the nightside oval can appear as multiple "curtains" (two, or more nearly parallel arcs; see, for example, early studies of Stormer, 1955; Nadubovich and Starkov, 1962; Davis, 1978), separated by distances ranging from several hundred kilometers to about one kilometer or even less (Davis, 1978; Maggs and Davis, 1968; Oguti, 1981; Velichko et al., 1987; Atkinson et al., 1989; Timofeev and Galperin, 1991; Borovsky, 1993). The multiplicity of arcs appears to be a rather typical phenomenon (often seen from the DMSP satellite images) for locally steady conditions within the auroral oval. Its importance for understanding of magnetospheric processes and mapping has been recognized for a long time (see Akasofu et al., 1967).

Field-aligned (FA) currents in multiple auroral arcs at the nightside have been measured several times in rocket auroral magnetic field experiments (Casserly and Cloutier, 1975; Sesiano and Cloutier, 1976) while, until recently, they were only noted rarely from satellite magnetometer measurements (see however, Lanchester and Wallis 1985; Bythrow et al., 1986; Potemra et al. 1987). In the 90s some prominent cases of multiple current sheets within the auroral oval were reported by Fukunishi et al., (1991); Obara et al., (1993) from AKEBONO satellite, Luhr et al., (1994) and Ohtani et al., (1994) from FREJA satellite.

Characteristic cases of paired medium-energy ions flux gradients in the dawn-dusk directions during late recovery phase (indicative of respective pressure gradients and hence to stratified FA current generation) were presented by Traver et al. (1994). At lower altitudes Volokitin et al. (1984), Girard and Senior, (1991), Yamamoto et al. (1993) and Opgenoorth et al. (1994) reported cases of small-and medium-scale stratifications of FA currents, fields and ionospheric parameters. 
Kotikov et al. (1993) in a systematic study of the filamentary structure ( $>60 \mathrm{~km}$ spacing) of the nightside westward electrojet, using ground-based data from the Kara chain of observatories $\left(157^{\circ} \mathrm{E}\right.$ magnetic longitude), noted its appearance when large-scale ionospheric conductivity and auroral riometer absorption are high, that is, the precipitation of electrons of more than $20 \mathrm{keV}$ is intensive and widespread.

The physics of auroral arc formation and the closely related problem of the arc thickness (but not specifically the arcs' multiplicity) were extensively reviewed and analyzed by Borovsky (1993) without selecting a unique mechanism for the auroral arc formation, particularly from the viewpoint of the wide spectrum of observed arc thicknesses.

We consider a case study of multiple narrow current sheet layering at an altitude of $2500 \mathrm{~km}$ on January 31 , 1990 from the magnetometer measurements of the MAGION-2 magnetically oriented Czech satellite (the subsatellite of the INTERCOSMOS-24 AKTIVNY satellite). They were launched in November 1989 to the polar orbit $(400 / 2500 \mathrm{~km}$ perigee/apogee, inclination $82^{\circ}$ ). MAGION-2 data were collected from December 21, 1989 till November 29, 1990. A similar case is briefly described from observations from the MAGION-3 satellite (the subsatellite of the INTERCOSMOS-25 APEX satellite).

The location of the field aligned current sheets, geophysical conditions of the pass 1563 on January 31 and inferred auroral effects suggest that the MAGION-2 crossed here the double oval, a remnant of the preceding substorms (see, Elphinstone et al., 1995), and detected a fine structure superimposed on the large scale architecture of the current sheets. The effects observed on pass 2057 on June 8, 1992 from the MAGION-3 and respective geophysical conditions were rather similar.

\section{MAGION-2 and MAGION-3 magnetometer experiments}

The Romanian triaxial fluxgate magnetometer $S G R-7$ (see, Ciobanu et al., 1993) was initially conceived as a navigation device for the magnetically oriented MAGION-2 and MAGION-3 subsatellites (Triska et al., 1990).

The magnetometer's sensors, oriented along the satellite's $\mathrm{X}, \mathrm{Y}$, and (magnetically oriented) $\mathrm{Z}$ axes, have two automatically switched measurement ranges: \pm 50048 $\mathrm{nT}$ and $\pm 6256 \mathrm{nT}$, with a resolution of $16 \mathrm{nT}$ for the first range and $2 \mathrm{nT}$ for the second one. The frequency range (at $-3 \mathrm{~dB}$ ) is $16 \mathrm{~Hz}$. An additional unit was a monoaxial variometer measuring magnetic induction fluctuations (Bv) along the $\mathrm{X}$ axis within the measurement range $\pm 156.4 \mathrm{nT}$. A fluxgate type sensor and a band pass amplifier give the quantization resolution of $0.05 \mathrm{nT}$; frequency range $0.1-20 \mathrm{~Hz}$; noise level $<0.1 \mathrm{nT}$.

The time resolution for both devices is limited by the telemetry rate (5 frames/s) to $100 \mathrm{~ms}$ for the DC measurements (2 values $\mathrm{B}_{\mathrm{x}}, \mathrm{B}_{\mathrm{y}}, \mathrm{B}_{\mathrm{z}} /$ frame) and $1 / 60 \mathrm{sec}$ for the variometer (12 values/frame). The telemetry data were received in real time at the Panska Ves station near Prague, Czech Republic.

\section{Data treatment procedures for the MAGION-2 pass 1563}

The direct subtraction of the IGRF 90 model values, (in order to assess the amplitude of the magnetic fluctuations), was not possible due to the uncertainty of the satellite's attitude, but the despinned polynomially fitted data trend was not too far from the model. Consistent levels of fluctuation (up to $300 \mathrm{nT}$ ) were observed on the components normal to the field line, suggesting that the satellite passed through a system of two medium-scale stratified bipolar field-aligned current bands, located at the edges of the double auroral oval and designated as the Poleward Band (POLB) and the Equatorward Band (EQUB). The small-scale layering of these two bands was observed as a higher frequency signal superimposed on the large scale trend (Fig. 1).

The FFT spectral analysis of the variometer's signal was performed using a $40 \mathrm{~s}$ window contiguously moved within the analyzed time interval (see Fig. 2). The CADR 3 program (Galperin et al., 1980) provided the geophysical coordinates and parameters needed along the pass from the orbital data.

Magnetometer and solar panel data showed a relatively smooth rotation of the satellite around $\mathrm{Z}$ axis with a period much greater than the structures' crossing period. This enabled us to determine the time-dependent transformation matrix with respect to the local magnetic field-aligned frame (MFA) as described by Luhr et al. (1994) (see Fig. 1).

Average inclination angles for the two medium-scale bands were determined by rotating the $\mathrm{X}_{\mathrm{MFA}}, \mathrm{Y}_{\mathrm{MFA}}$ axes around the $Z_{\mathrm{MFA}}$ axis, by one degree step, until the minimum of the $\sum_{i=1}^{n}\left(\mathrm{~B}_{\mathrm{xMFA}}\right)^{2}$ was reached (Figs. 3a, 4a), $n=$ number of measurements. The minimization, done separately for each band, provided average inclination angles (counterclockwise from the E) of $-25 \pm 12^{\circ}$ for the POLB and $32 \pm 12^{\circ}$ for the EQUB. The EQUB current sheets were less regular than those in the POLB, indicating larger deviations from parallelism or time variations. So the latter value is less certain for the average inclination. It can be noted that this band apparently was located close to the Harang discontinuity. These two factors are important to note for an explanation of the non-parallelism of the EQUB and POLB.

The derivative $d B_{\text {Yrotated }} / \mathrm{dX}_{\text {rotated }}$ was then calculated and averaged over five sequential values, providing the current density profiles, computed using Ampere's law under the supposition of infinite steady parallel current sheets (Figs. 3b, 4b).

\section{Multiple current sheets on pass 1563 of the MAGION-2}

The Kiruna magnetic data at this time in the evening sector showed that some activity in the form of an 
Orbit 1563 MAGION-2 (31.01.1990)

(17:56:02 UT-18:03:32 UT)

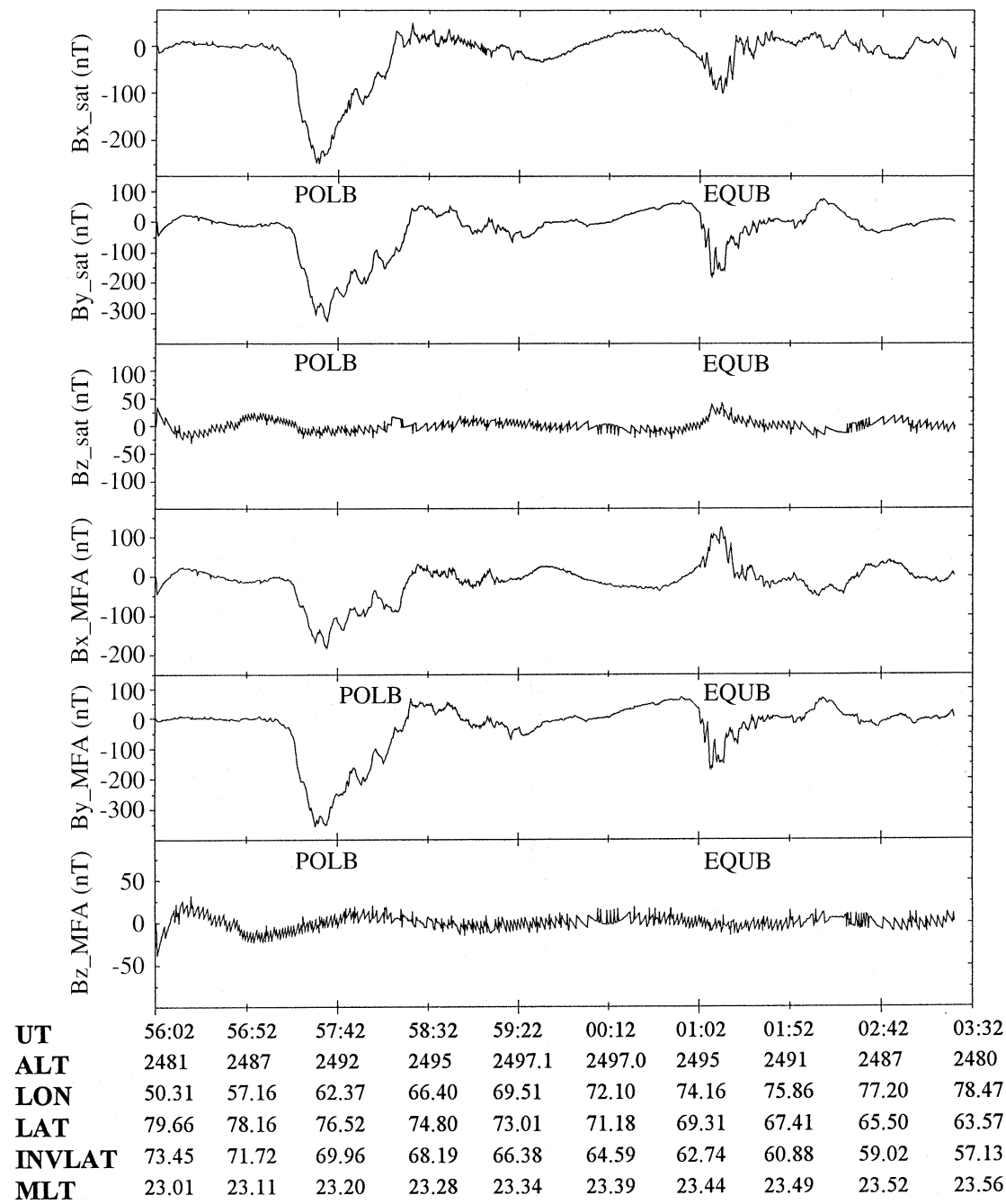

Fig. 1. Magnetic field components, Bx, By, Bz, of field aligned currents (satellite and MFA frame). Two double-sheet current bands (POLB and EQUB, respectively, can be seen with weaker currents in between. The upward current branch of each band is stratified into several narrow double-sheet current filaments shown in detail in Figs. 3, 4

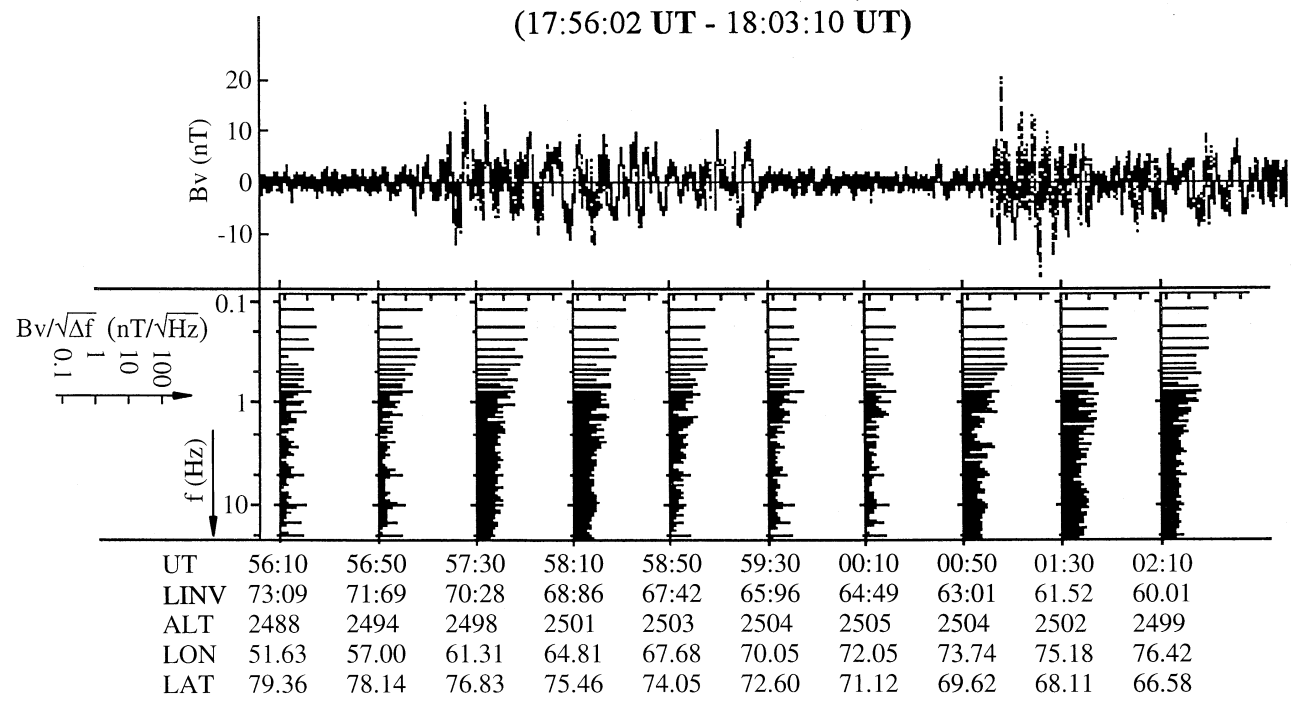

Fig. 2. Magnetic field variation measured by the SGR- 6 variometer, oriented along the satellite $\mathrm{X}$ axis (upper panel). The FFT spectral density calculated for a $40 \mathrm{~s}$ contiguously moving window is also shown (lower panel). The POLB and EQUB small-scale features are visible as enhancements of the spectral density between $0.1-0.2 \mathrm{~Hz}$ 
Orbit 1563 MAGION-2 (31.01.1990)

(17:57:02 UT - 17:58:42 UT)

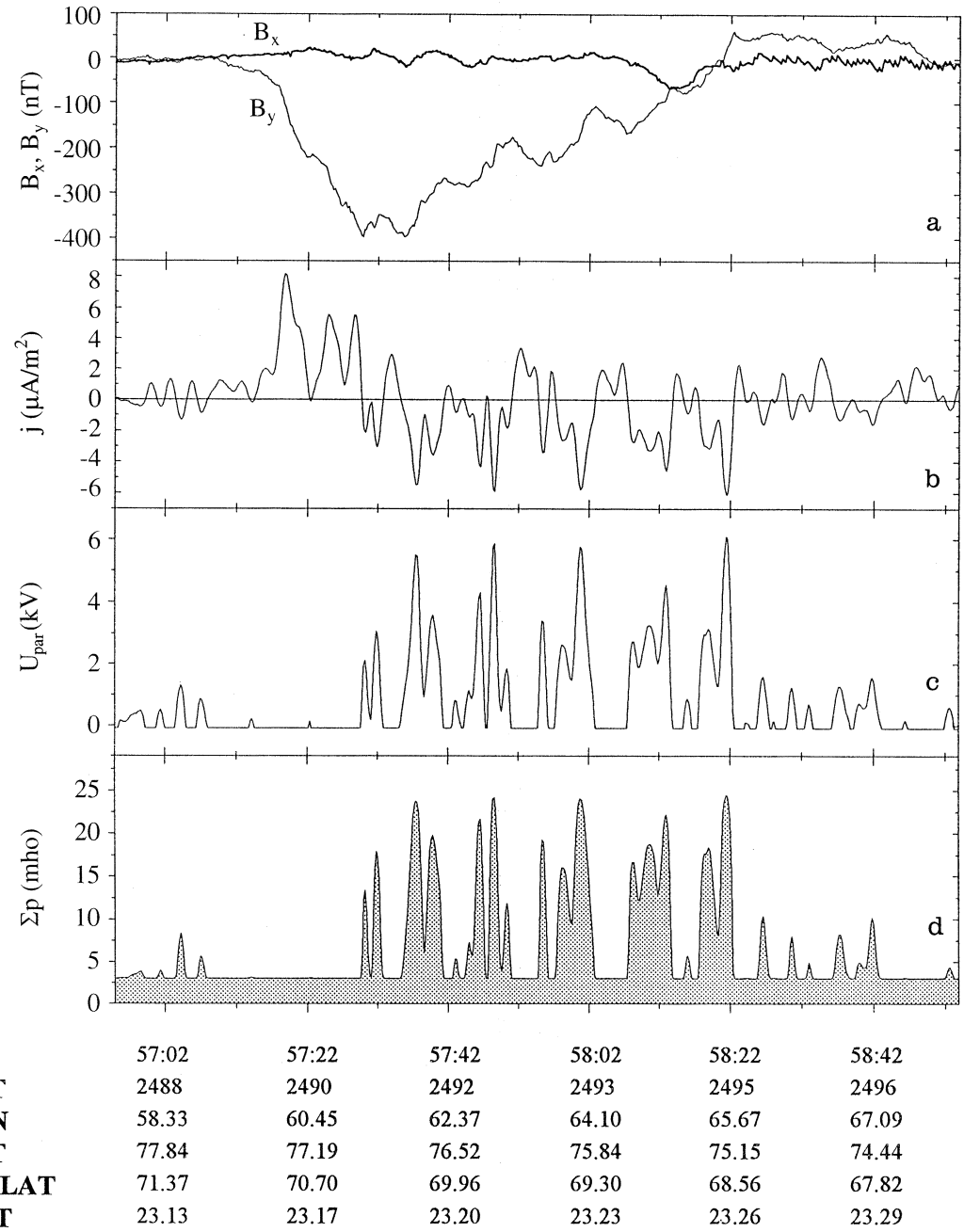

Fig. 3. a Magnetic field components in the POLB's principal axis reference system; $\mathbf{b}$ field aligned current density (positive for downward current); c resulting field aligned potential drop (Lu et al., 1991; Galperin et al., 1992); d resulting Pedersen conductivity (Galperin et al., 1992) isolated substorm ceased by 16:00 UT, and the time interval 16:00-18:00 UT was quiet. Soon after 18:00 UT an early growth phase started at this meridian while the magnetometer data of the same day of the Sodankyla station (in between Kiruna and the Kara Sea chain) showed the start of an activation, or a weak substorm activity, after $18 \mathrm{UT}$.

Magnetometer records from the Kara Sea chain, located approximately below the pass, (Fig. 7) are shown on Fig. 5. A weak disturbance of about $100 \mathrm{nT}$ occurred just around $18 \mathrm{UT}$, the time of pass 1563, and it was more pronounced at the equatorial part of the oval. It appeared to be connected to this activation, or a localized weak substorm, observed at Sodankyla. At 18:55 UT a notable substorm onset of about $700 \mathrm{nT}$ occurred at latitudes lower than the Izv (IZV) magnetic observatory (INVLAT $=70.25$ ) close to this meridian. Thus the time of the MAGION-2 pass 1563 along the midnight meridian can be considered as the time close to an activation, or a weak substorm, localized in the premidnight sector. This occurred after two hours of recovery from the preceding intense substorm, or at the time of an early growth phase of the next intense substorm during a disturbed day. This is consistent with the variations of the precipitation boundaries as measured from the DMSP satellites around the time of the MAGION-2 pass (see later).

Calculation of the average spacing (projected to the auroral altitude $\sim 100 \mathrm{~km}$ ) of the narrow current sheets, across the current bands, gives about $35 \mathrm{~km}$ for the 4 small-scale sheets of POLB and $15 \mathrm{~km}$ for the EQUB's arcs (not so clearly defined).

Additional information about medium-and smallscale/high-frequency magnetic field variations comes from the analysis of the variometer's AC measurements (See Fig. 2). An increase of power in the AC channel $(0.1-20 \mathrm{~Hz}$ ) started at 17:57:20 UT (when POLB was identified in the DC components) with the maximum of power density located at $0.1-0.2 \mathrm{~Hz}$ frequency, which converted from the temporal to the spatial scale corresponds to a latitude spacing period of about $30-40 \mathrm{~km}$ at auroral altitude. A comparison between the wave phase and the derivative of DC $B_{Y}$ component is consistent with the field variations produced by a DC current modulation. A second increase of the wave power started around 18:01:05 UT (when EQUB was 
Orbit 1563 MAGION-2 (31.01.1990)

(18:00:42 UT - 18:03:12 UT)

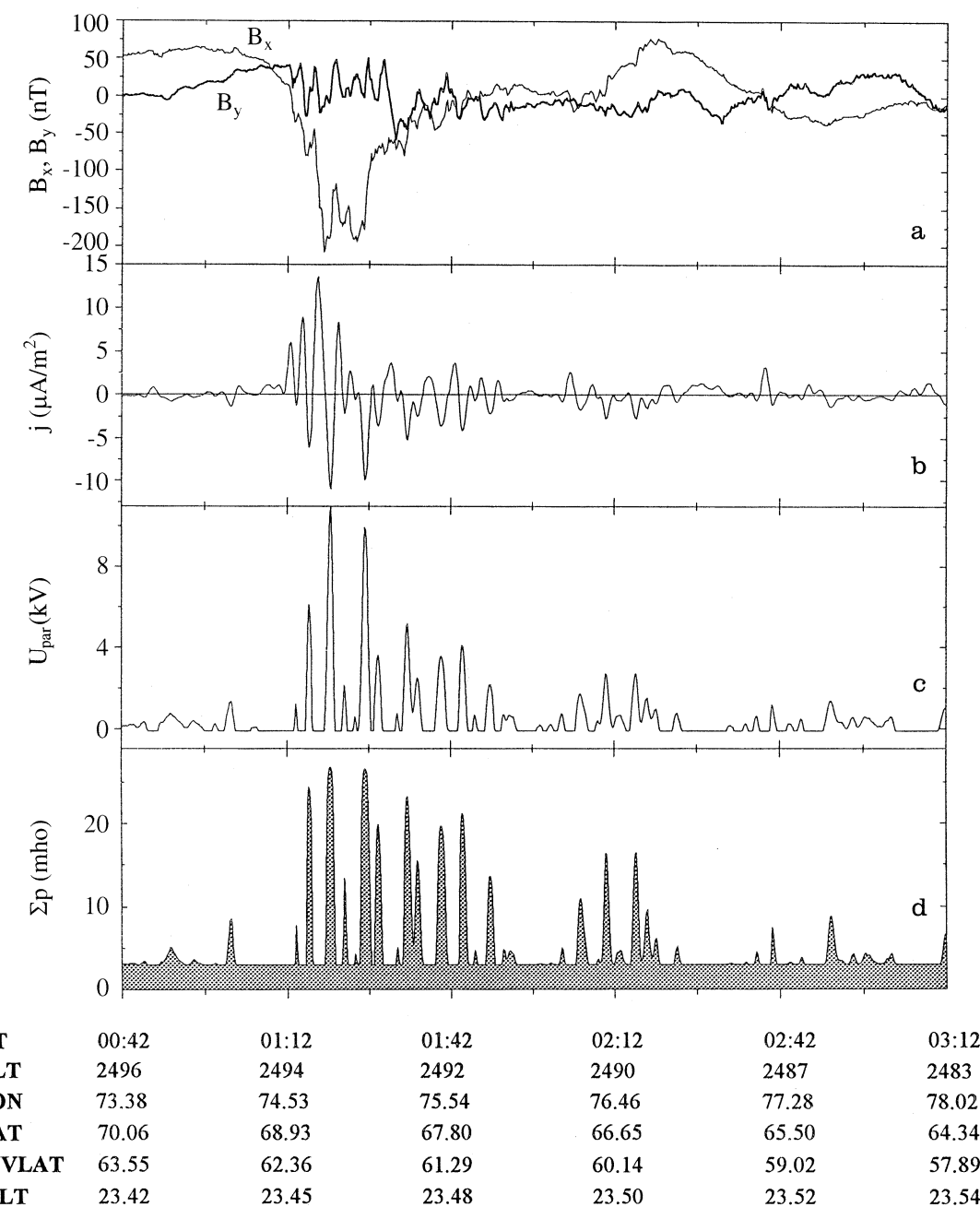

Fig. 4. a Magnetic field components in the EQUB's principal axis reference system; b field aligned current density (positive for downward current); c resulting field aligned potential drop (Lu et al. 1991; Galperin et al., 1992); d resulting Pedersen conductivity (Galperin et al., 1992) identified) with the maximum of power density registered at the higher frequency. For this band the wave phaseDC $B_{Y}$ correlation is not so good as for the POLB.

\section{Multiple current sheets on pass 2057 of the MAGION-3}

A similar case of a double oval with a layered polar and equatorial double sheet FA current bands separated by a wide region with very weak currents were found from the data of the MAGION-3 satellite.

The midnight MAGION-3 pass 2057 at 21 UT on June 8, 1992 occurred just above the Kiruna Observatory (see Fig 6, which uses the same format as Fig. 1, except variometer data which was not available). According to the Kiruna magnetogram this was a period of constant agitation without appreciable electrojets within a large magnetic storm on June 7-9, 1992.

Satellite data showed a rather homogenous downward FA current branch $60 \mathrm{~km}$ wide, located at the polar side of the polar band (INVLAT 67.1-67.8). The adjacent upward FA current branch (also $\sim 60 \mathrm{~km}$ wide) of the polar band at its equatorial side (INVLAT 66.2-67.1) was stratified in four narrow double current sheets. Equatorward from this polar FA current band there was a region without appreciable FA current, and then, in the region of INVLAT 60.0 - 64.4 another FA current band was observed. The whole FA current structure on this pass was consistent with the double oval and was very similar to the structure observed on the MAGION-2 pass 1563 already discussed in detail.

A polar FA current band separated from the equatorial band of strong FA currents was also crossed $2 \mathrm{~h}$ later on pass 2058. It resembled in shape the polar band crossed on pass 2057, but was nearly twice as wide and shifted equatorward by about $2^{\circ}$. With respect to the observation from pass 2057, the equatorial FA current band on this pass showed a significant difference. It was crossed at the time of the onset of a substorm at Kiruna which occurred at the end of the quiet interval. The data from this pass will not be analyzed further here. 
08.06 .1990

17:00 UT-20:00 UT

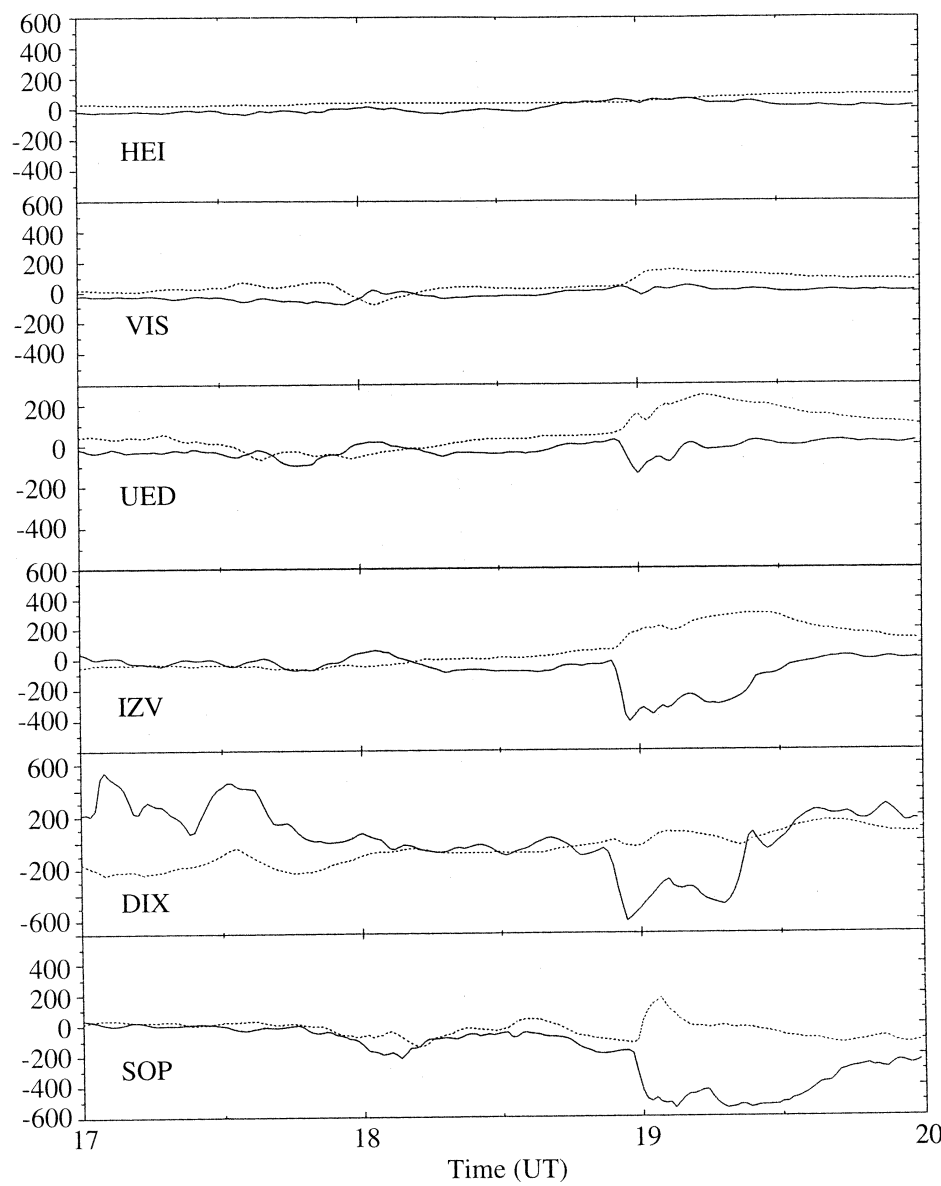

Fig. 5. The KARA chain of magnetic observatories magnetograms $(\mathrm{H}$, solid line; $Z$ dotted line $)$ arround pass 1563 of the MAGION-2 above the chain at about 18:00 UT on January 31, 1990. The magnetic observatories from top to bottom are : Heiss

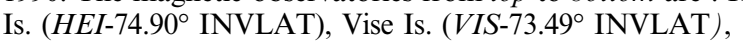

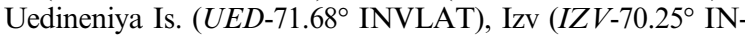
VLAT), Dixon Is. (DIX-68.20 INVLAT), Sopochnaya Karga (SOP-66.68 INVLAT)

\section{Inferred and observable auroral and precipitation effects}

As is known, a DC upward current of sufficient amplitude, that is, an intense downward flux of precipitating energetic electrons, will produce a FA potential drop which results in auroral electron acceleration, auroral excitation and ionization, and a modification of the electric field pattern (see Kan 1982; Lyons 1992; Galperin, 1994a). For calculations of the effects of the auroral FA currents we modified the program developed by one of the authors (Galperin 1992; Galperin et al., 1992) using as inputs the current density deduced for POLB and EQUB (Figs. 2c-e 3c-e).

The field-aligned potential drop calculated with the empirical formulas using Lyons-Evans-Lundin constant $\mathrm{K}(\mathrm{LEL})=10^{-9} \mathrm{Mho} / \mathrm{m}^{2}$ reached values of $4-6 \mathrm{kV}$ in the POLB (Fig. 3c) and somewhat larger (6-11 kV) for the EQUB (Fig. 4c). Strong Pedersen conductivity enhancement was inferred from the same model, reaching values of 20-25 Mho in the most disturbed zones (Figs. 3d, 4d). Qualitatively this is consistent with typical conductivity values measured in discrete arcs using radar backscatter techniques (Horwitz et al., 1978).

Clearly the rather intense narrow current sheets on pass 1563 must produce very strong auroral effects in the ionosphere, easily observable from ground-based stations or satellites if they happened to register the data at the time.

The locations of precipitation boundaries for the period around pass 1563 were taken from the automated DMSP database constructed by Newell et al., (1991). Three passes of the DMSP satellites in the near-midnight sector were taken for comparison (See Fig. 7). The first one (DMSP-A) occurred at 23.2 to 0.6 MLT on 17:2417:27 UT, i.e. half an hour before the MAGION-2 pass 1563. The second one (DMSP-B) was at 19.9 to 21.6 MLT on 18:49 to 18:52 UT, about 50 minutes after the pass 1563 , just before the substorm onset at the Kara chain. The third one (DMSP-C) was at 22.6 to $0.3 \mathrm{MLT}$ on 19:06-19:11 UT, i.e. at the time of the substorm expansion phase but at a slightly earlier local time sector. The equatorial boundary of discrete auroral precipitation on pass DMSP-A was located at $65.8^{\circ}$ invariant latitude while the latitude of the EQUB equatorial edge was about $61.2^{\circ}$. On pass DMSP-B this boundary was at $66.1^{\circ}$, i.e. it hardly moved, and on pass DMSP-C it was still at $65.6^{\circ}$. The polar boundary of the discrete electron precipitation was at $>72.9^{\circ}$; at $72.4^{\circ}$ and at $>71.0^{\circ}$ invariant latitude on passes $\mathrm{A}, \mathrm{B}$ and $\mathrm{C}$ respectively, to be compared with the polar edge of the upward part of POLB at $70.3^{\circ}$. On passes $\mathrm{B}$ and $\mathrm{C}$ a layering is observed 
Orbit 2057 MAGION-3 (8.06.1992)

(21:01:07 UT - 21:05:00 UT)

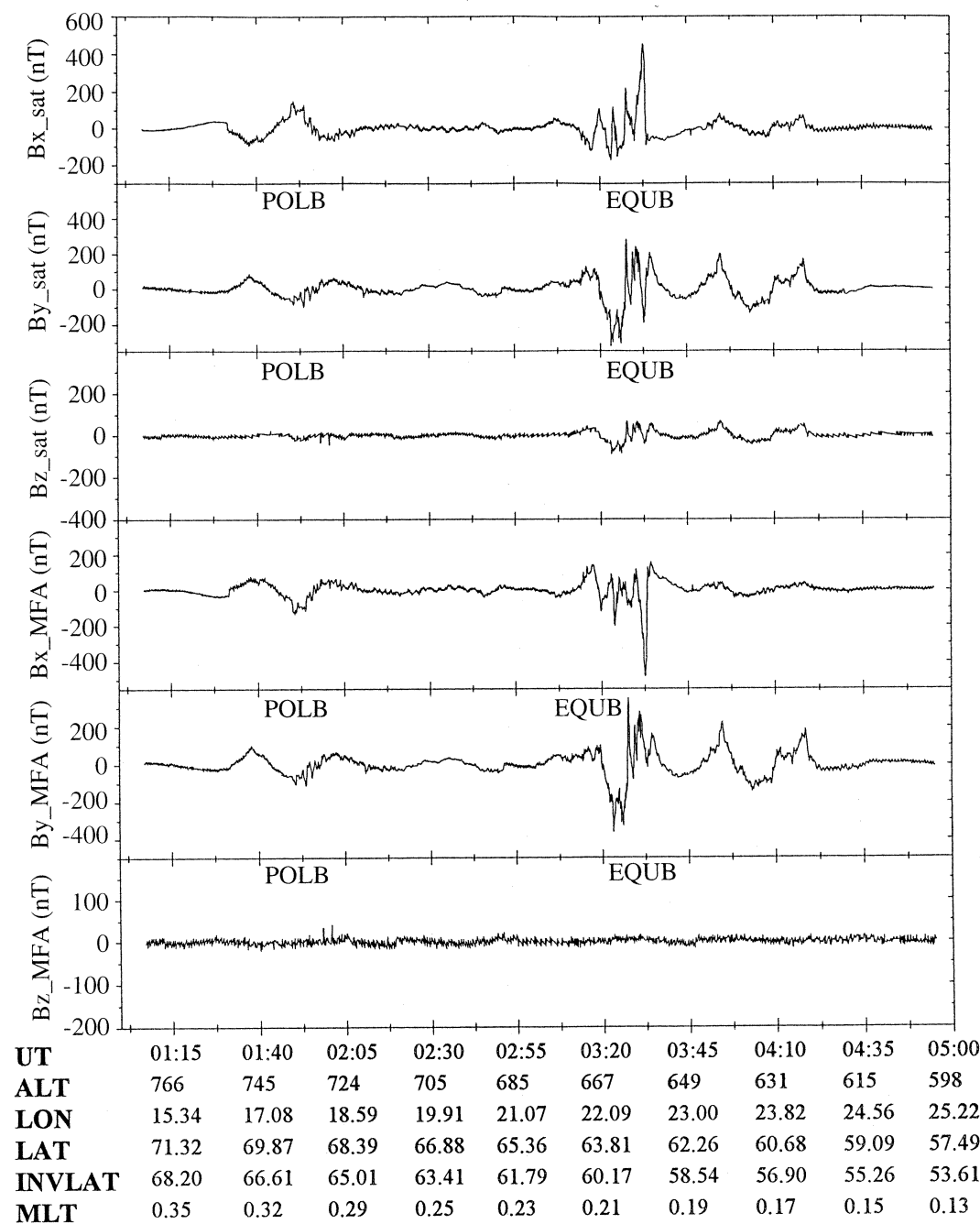

Fig. 6. Magnetic field components of field aligned currents (satellite and MFA frame) observed for MAGION-3 satellite. Two large-scale current system (POLB and EQUB) with superimposed small-scale structures are observed of the discrete precipitation zone from the DMSP data to two distinct precipitation bands on $\mathrm{B}$ and to three on $\mathrm{C}$ (Fig. 7). The occurrence of layered FA current bands southward in the case of MAGION-2 pass could be explained by the fact that these measurements were taken at different times (about 30-60 min in respect of DMSP passes) and during a period when two successive passes of DMSP (A and C) showed significant changes. Also, due to the relatively high current densities and to the specific spatial scales involved in the MAGION-2 measurements, the observed micro-scale stratification could have only a localized extension not seen at the DMSP trajectories.

For the DMSP data during the MAGION-3 passes 2057 and 2058, the boundaries' locations are available only in the dawn-dusk meridian from 20:00 till 23:30 UT on June 8, 1992. Generally they show relative stability of the precipitation boundaries both for diffuse and discrete precipitation. An exception is the appearance of a strong discrete precipitation feature crossed at 21:16:41-21:19:25 UT in the range 68.0-76.0 INVLAT at 16.9-16.2 MLT by the DSMP 11 satellite. This intense FA precipitation at the dusk possibly could be connected to the appearance of a very intense FA current band crossed at midnight by the MAGION-3 satellite on the pass 2057 at 21:03:30 UT at around INVLAT 60.0. As already described, no corresponding magnetic activity was noted below the pass at Kiruna (located about $5^{\circ}$ northward from the intense FA current band).

At the post-midnight sector at 2.0-3.0 MLT a structured (or, discrete) electron precipitation between the 2057 and 2058 passes was seen by the DMSP 11 satellite at 22:07 UT in the band 66.7 to 73.2 INVLAT. By 23:46 UT, about $40 \mathrm{~min}$ after the 2058 pass, the equatorial boundary of the structured precipitation in this MLT sector had descended to 64.9 INVLAT.

There is an important difference in space and time when comparing these DMSP data with the currents measured at the midnight meridian from the MAGION3 on the passes 2057-2058. However, these DMSP data are consistent with the inference made already about the stability of the oval during the recovery period when the double-oval FA current structure was observed on pass 2057. 

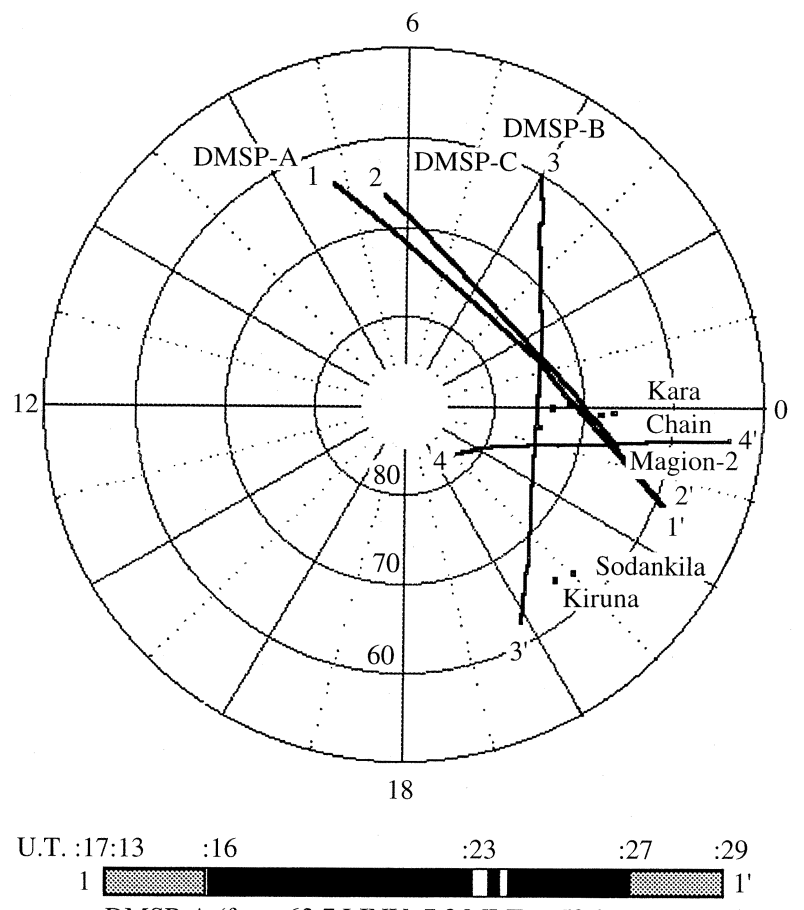

DMSP-A (from 63.7 LINV, 7.2 MLT to 59.2 LINV, 22.6 MLT)

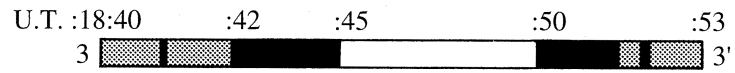

DMSP-B (from 60 LINV, 4.0 MLT to 62.4 LINV, 19.9 MLT)

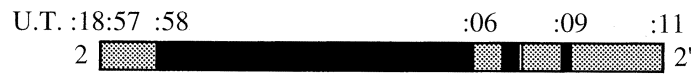

DMSP-C (from 66 LINV, 6.4 MLT to 59 LINV, 22.6 MLT)
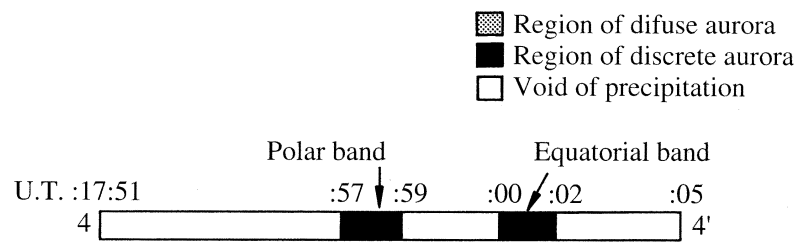

Magion-2 (from 82.1 LINV, 21.2 MLT to 53.8 LINV, 23.6 MLT)

Fig. 7. DMSP orbits (A between 17:13-17:29 UT, B between 18:4018:54 UT, between 18:57-19:11 UT) and MAGION-2 orbit (between 17:51-18:05 UT) and the observed pattern of precipitation above the northern auroral oval (in MLT-LINV coordinates)

\section{Discussion}

\subsection{Field-aligned current sheets stratification during quieting}

For the geophysical conditions described already for pass 1563, the appearance of multiple parallel arcs/ current sheets seems to be common (see Davis, 1978). However their large quantity (4 arcs or more in one band), small-scale spacing (15-35 km) and the simultaneous layering of the auroral oval to two widely separated striated bands located at the poleward and equatorward edges of the oval is apparently a unique case from the 600 MAGION-2 analyzed orbits. A preliminary search in the MAGION-3 data revealed on pass 2057 a similar double-oval case with upward FA current branches stratification both in the polar and equatorial FA current bands during a quieting period within the magnetic storm of June 7-9, 1992. While still a rare occasion, the similarity of the double-oval FA structure between these two cases allows us to suggest that FA current layering could be a feature of the auroral structures during double-oval conditions.

The observed narrow upward field-aligned sheet currents we identify here with the quasi-DC multiple auroral curtains (arcs). They are contained mostly within a medium-scale upward field-aligned current branch of the double-sheet current band. Each of the two medium-scale bands, is supposed to be a wide inverted-V structure with superimposed smaller scale quasi-periodic double-sheet current structures (microscale (high-frequency) FA currents and/or Alfven waves). Such a current stratification is consistent with the Matreshka scheme of encircled current loops (double-sheet current pairs) of different spatial scales, and parallel direction of the closing ionospheric current, which are characteristic for steady auroral conditions (see Timofeev and Galperin, 1991). The current balance (upward versus downward) was achieved at the medium-scale level, while the current integrated across the small-scale sheets looks rather unbalanced, since the integrated upward current is about 100\% larger. However the data treatment method used (approximation of the undisturbed level by a polinomial) may influence this evaluation of balance, so its significance is doubtful.

The two cases of double-oval FA currents described here have many similarities, but also some important differences, to the cases of multiple current sheet crossings by the FREJA satellite as described by Ohtani et al., (1994) and Luhr et al., (1994). The later cases occurred in the post-midnight sector also during a recovery phase and consisted of periodic current filaments (multiple double current sheets) separated by distances of 200 to $400 \mathrm{~km}$ (Ohtani et al., 1994) and $50 \mathrm{~km}$ (Luhr et al., 1994), accompanied by small-scale currents/Alfven waves as seen from the AC channels on both cases. The spacing periods and geophysical conditions of these observations (the quieting after an important disturbance) are comparable to the MAGION-2 and MAGION-3 cases considered here.

It follows from comparison of all these cases that a wide spectrum of spacing is possible in the multi-layer current sheets which is consistent with similar results on auroral arcs (Davis, 1978). However, no periodic variations were observed at the ground magnetic observatories along the MAGION-2 satellite track and this could be due to the small separation of the current sheets, and/or to the lack of their latitudinal drift in the respective cases.

We can conclude that the multiple FA current sheet / multiple arcs phenomenon is not uncommon for doubleoval conditions of the auroral oval quieting following a strong disturbance. The spacing between the current sheets / arcs were observed over a range of $\sim 15 \mathrm{~km}$ to $\sim 400 \mathrm{~km}$ from various satellites. 
According to contemporary views on the mapping problem, the EQUB is projected to the inner edge of the plasma sheet at about 5-15 Re (see Feldstein and Galperin, 1985; Galperin and Feldstein, 1991; Pulkkinen et al., 1993; Lyons and Samson, 1992). The mapping of the POLB is not quite certain especially during such a quieting interval within a rather disturbed period. Most probably the POLB maps somewhere close to, or at, the separatrix formed in the mid-tail or distant tail during a recent disturbance (see Galperin, 1995; Galperin and Feldstein, 1996; Pulkkinen et al., 1995). Thus, the projections of the stratified medium-scale current bands, POLB and EQUB, to the tail plasma sheet are to be located at a large distance one from another in the radial direction along the tail.

An indirect support to the last supposition can be derived from the data of pass 2058 of the MAGION-3. This pass occurred during a substorm at Kiruna. It affected the FA currents of the equatorial band crossed at INVLAT 55.4-59.7, but not the currents of the polar band at INVLAT 61.6-65.1, which remained rather similar in shape (though widened and shifted equatorward about $2^{\circ}$ ) to that crossed on the preceding pass 2057.

Thus, the stratification of the upward current sheets during the quieting period had to proceed more or less simultaneously in these two spatially separated FA current generating regions of the tail.

Next we consider possible mechanisms of the FA current sheet stratification.

\subsection{Sheet current stratification due to ionospheric effects}

The mechanism of multiple arc generation by Alfven wave resonance in the ionospheric Alfven resonator (IAR) at the altitude range of $1-2 R_{E}$ was originally suggested by Atkinson (1970) and described in detail by Trakhtengertz and Feldstein, (1984, 1987a, b, 1991) and Lysak, (1990, 1991). It is unlikely to be consistent with the observed spacing because the respective Alfven waves with $\mathrm{k}_{\perp}^{-1}$ of more than $\sim 8-10 \mathrm{~km}$ according to Forget et al., (1991) will not be trapped in the IAR, and these waves will reach the plasma sheet. Thus, a plasma sheet stratification both at its near-Earth edge and at its distant part (presumably close to the separatrix) is inferred from these observational data.

At the same time, FFT spectra for this period show a strong increase of wave power which, if considered as spatial, gives scales of the order of those predicted by the IAR theory. Thus, the data do not exclude effects of small-scale wave trapping in the IAR as a secondary process.

According to contemporary M-I coupling models the appearance of multiple arcs is a characteristic feature (see Kan et al., 1988) related to Alfven waves reflections from the plasma sheet (Watanabe et al., 1993) or to magnetospheric shear flows (Zhu et al., 1993) both considering a non-linear interaction with the ionospheric "load". Earlier versions of the M-I coupling model (Sato, 1978) reached the conclusion that the main factor leading to the arcs' multiplicity is the difference in time constants between the Alfven wave bouncing and the recombination in ionosphere subject to the nonlinear and time-delayed conductivity response to the induced auroral precipitation. In Zhu et al's, 1994 study for example, the ratio $\mathrm{R}$ of Hall-to-Pedersen conductivity (related to the mean auroral electron energy) determined the arc spacing which changed from 60 to $100 \mathrm{~km}$ for R from 1.0 to 2.0, while the quantity of arcs increased from one to five with the change in electric field from 20 to $30 \mathrm{mV} / \mathrm{m}$.

For the case of the current sheet multiplicity on the pass 1563 of the MAGION-2, the DMSP precipitation data imply that during the preceding disturbance the average conductivity level in the nightside oval increased significantly and remained enhanced during the quieting period when pass 1563 occurred. As was suggested by Kotikov et al., (1993) these conditions statistically favor the current sheet stratification, and qualitatively it is consistent with the M-I coupling model calculations described above.

\subsection{Sheet current stratification in the plasma sheet current source}

A steady elongated generator of a double current sheet (downward and upward) in the plasma sheet, where the EQUB is mapped along geomagnetic field lines, was considered in a model of double field-aligned (FA) current sheet by Galperin, (1992) and Galperin et al., (1992). It was based on a phenomenological inclusion of non-adiabatic ion motion effects (deterministic chaos) in the neutral sheet accompanied by an enhanced narrow cross-tail westward "line current". The narrow magnetic field minimum produced by the line current along the neutral sheet inner edge was considered the "arc's (FA current) root".

These considerations are qualitatively consistent with experimental data on ion precipitation and extensive numerical ion trajectory calculations (see Bosqued et al., 1992; Ashour-Abdalla et al., 1993, 1994). They lead to the conclusion that the location at least of the most equatorward arc in the premidnight sector is intimately connected with the global plasma sheet structure. At the same time it is not straightforward to extend this single arc generation model (as well as the model by Rothwell et al., 1991), to multiple arc formation, as was observed in EQUB.

It was noted that the layering within the POLB (and mostly also within the EQUB) occurs in the upward current branch of the double-sheet band. This is qualitatively consistent with the theory of Tverskoy, (1982a, b) according to which a positive feedback exists for a small perturbation of the FA upward current density (but not for the downward current, too). It predicts a dimensionless parameter $G$ defining the quantity of the adjacent current sheets:

$$
G=\frac{e *[E] * L}{T_{1}}=\frac{e * j_{\text {parall }} * L^{2}}{\Sigma_{p} * T_{i}}
$$


where $e=$ electron charge; $[E]=$ electric field jump across the arc; $T_{i}=$ plasma sheet ion temperature; $L=$ half-thickness of the upward current band, $j_{\text {par }}$ all $=$ maximum upward FA current density, and $\sum_{p}=$ average Pedersen conductivity in the whole auroral band. For ranges of $G \sim 1 ; \sim 6$; and $\sim 30-40$, the predicted quantity of arcs (or inverted-Vs) was 1 , or 2 , or 3, respectively. This prediction (called "hot stratification") was developed further in Antonova et al., (1988). It was tested using the data of the INTERCOSMOS-Bulgaria-1300 satellite by Antonova et al., (1991, 1993). The results of the test, though rather sparse statistically, look very favorable. For the $G$ value ranges $1-3,8-9$ and $18-40$, there were indeed one, two or three inverted-Vs observed from the particle data.

If we take for the double-sheet POLB the measured total width $L=100 \mathrm{~km}$, and $j_{\text {parall } \max }=10 \mu \mathrm{A} / \mathrm{m}^{2}$, and estimated values $\Sigma_{p}=2$ Mho, $T_{i}=4 \mathrm{keV}$, we produce a $G$ of 12 . The two values in the denominator of the formula are rather arbitrary because there are no particle data on pass 1563. The ionospheric E-layer was in darkness, but the DMSP precipitation data indicate appreciable precipitation before and after pass 1563. Thus the error in the assumed value of $\Sigma_{p}$ can be estimated by a factor of 2, and similar error can be in the assumed value of $T_{i}$. Thus, the total range of $G$ can be estimated from 6 to 50 . From the linear theory such values of $G$ can indicate that some layering (to two or more sheets) of the upward current band is implied.

We have already suggested that the location of the POLB could be coincident with the most poleward arc of the double oval (Elphinstone et al., 1995). It is not quite clear where the current source for this structure is located in the distant tail, and what is its nature. It may be speculated that the origin of the current sheets within the POLB are a composite of X-lines and intervening plasmoids that were launched downtail during earlier substorm activity bursts. Multiple X-lines are a possible source of multiple auroral arcs as was suggested by Atkinson et al., (1989). Their spacing in this case could be a result of particular relative locations of the X-lines and of the mapping geometry. Such a supposition is consistent with the observed absence of a preferable spatial scale for the arc spacing.

\subsection{Non-linear standing Alfven waves in the $\mathrm{mHz}$ range and multiple arcs}

As shown by J. Samson and his group, both from observational and theoretical grounds (see Samson et al., 1992; Samson and Rankin, 1994; Rankin et al., 1994; Liu et al., 1995), non-linear standing lowfrequency waves in the $\mathrm{mHz}$ range can be intimately related to the arcs' formation and their multiplicity. In this theory the resonances are fed by broad-band noise in the system, and it was shown that auroral electrons accelerated by FA electric field due to large $\mathbf{k}_{\perp}$ in the Alfven wave can reach typical auroral energies in a steady state. The energy flux, width and spacing in a multiple arc system are explainable using this approach which considers the plasma sheet and ionosphere as a strongly coupled system. This theory and its further developments is a way to combine several preceding approaches. Some of them were discussed earlier for near and middle tail which corresponds to the equatorial and central parts of the oval. However it is hard to see how to apply this fruitful concept to the polar part of the oval which apparently implies too long periods for a resonance.

\section{Conclusions}

As was indicated by Akasofu et al., (1967) the multiplicity of auroral arcs may carry important information about stratification processes in the tail. Despite very significant progress during recent years in various special domains of magnetospheric physics, these processes still remain largely unexplained. It seems that the current sheet layering in the near and middle tail could differ from that in the distant tail, while their ionospheric signatures, the multiple arcs, are quite similar. However, comprehensive theories and numerical models unifying particular local mechanisms in a global magnetospheric / ionospheric perspective are only beginning to appear in the literature. Thus, the two case studies described, which occurred in the double-oval conditions, as well as other similar cases, could be of interest for comparisons with theories and model calculations of magnetospheric relaxation after a substorm.

Even conditions and physical reasons of the arc multiplicity development are still not established firmly. Partly it is a result of scarcity of observational data due to the limited resolution of space UV imagers. This is because of the optically thick emission layer in Oxygen resonance lines at $130 \mathrm{~nm}$ which do not allow to resolve spacing of less than about $50 \mathrm{~km}$. Calculations of expected auroral / ionospheric effects presented here for the currents observed on pass 1563 of MAGION-2 are aimed at providing characteristics which could be used in the further modeling of the magnetosphere during quieting periods. It is hoped that such ground-based arrays as CANOPUS in Canada and the combined array in Scandinavia, Svalbard and Kola Peninsula will provide needed detailed experimental information on these topics which will make it possible to compare them with the data from the constellation of high-altitude satellites GEOTAIL, INTERBALL, POLAR and CLUSTER and those from low-altitude satellites in the next few years.

Acknowledgements. We thank Pavel Triska, the PI of the MAGION-2, and the entire team of participants, for advice and help rendered during the experiment and data treatment procedures. We thank Pat Newell for his help with the DMSP data base use. We would like to thank the International Science Foundation and the Russian Foundation for Basic Research for the support of this work for YIG, NVJ and TMM by the grants ISF N7S000 and RFBR 94-02-04299a and for ALK, EMS and OAT by the grants ISF NSG000 and RFBR 95-05-15327a.

Topical Editor K.-H. Glaßmeier thanks R. Elphinstone and T. Pulkkinen for their help in evaluating this paper. 


\section{References}

Akasofu, S.-I., S. C. Chapman, and P. C. Kendall, The significance of the multiple structure of the auroral arc, in: Aurora and Airglow, Ed. B.M., McCormac, Reinhold Publ. Corp., New York, Amsterdam, London, 281-286, 1967.

Antonova, E. E., V. I. Lazarev, M. V. Stepanova, B. A. Tverskoy, M. V. Teltsov, A. K. Kuzmin, S. I. Shkolnikova, and S. I. Isaev, Parameters of the multiplet structures of the inverted-V type according to the Intercosmos-Bulgaria-1300 satellite data, Geomagn. Aeron. (in Russian), 31, 258-267, 1991.

Antonova, E. E., M. V. Stepanova, M. V. Teltsov, and B. A. Tverskoy, Characteristics of multiple auroral Inverted-V structures and the problem of magnetospheric plasma stratification, Preprint INP MSU-93-4/295, 1-32, 1993.

Antonova, E. E., M. V. Stepanova, and B. A. Tverskoy, The influence of self-consistent ionospheric conductivity on the fieldaligned currents stratification, Geomagn. Aeron. (in Russian), 28, 16-21, 1988.

Ashour-Abdalla, M., J. P. Berchem, J. Buchner, and L. M. Zelenyi, Shaping of the magnetotail from the mantle: global and local structuring, J. Geophys. Res., 98, 5651-5676, 1993

Ashour-Abdalla, M., L. M. Zelenyi, V. Peroomian, and R. Richard, Consequences of magnetotail ion dynamics, J. Geophys. Res., 99, 14891-14916, 1994.

Atkinson, G., Auroral arcs: result of the interaction of a dynamic magnetosphere with the ionosphere, J. Geophys. Res., 75, 4746$4755,1970$.

Atkinson, G., F. Creutzberg, R. L. Gattinger, and J. S. Murphree, Interpretation of complicated discrete arc structure and behavior in terms of multiple X-lines, J. Geophys. Res., 94, 5292-5302, 1989

Borovsky, J. E., Auroral arc thicknesses as predicted by various theories, J. Geophys. Res., 98, 6101-6138, 1993.

Bosqued, J. -M., M. Ashour-Abdalla, L. M. Zelenyi, A. Berthelier and Y. I. Galperin, Intensification of cross tail current by stochastic processes, EOS, 73, Suppl., Abstracts, 260, 1992. (Abstr.).

Bythrow, P. F., M. A. Doyle, T. A. Potemra, L. J. Zanetti, R. E. Huffman, C. -I. Meng, D. A. Hardy, F. J. Rich, and R. A. Heelis, Multiple auroral arcs and Birkeland currents: evidence for plasma sheet boundary waves, Geophys. Res. Lett., 13, 805-808, 1986.

Casserly, R. T., Jr., and P. A. Cloutier, Rocket-based magnetic observations of auroral Birkeland currents in association with a structured auroral arc, J. Geophys. Res., 80, 2165-2168, 1975.

Ciobanu, M., Georgescu E., Moldovanu, A., Popescu, D., Comisel, H., Macoviciuc, M., Catalog of Auroral Geomagnetic Events Observed by the MAGION-2 Satellite, Ed Institute of Space Sciences Bucharest, 1993.

Davis, T. N., Observed characteristics of auroral forms, Space Sci. Rev., 22, 77-113, 1978.

Elphinstone, R. D., J. S. Murphree, D. Hearn, L. L. Cogger, I. Sandahl, P. T. Newell, D. M. Klumpar, S. Ohtani, J. A. Sauvaud, T. A. Potemra, K. Mursula, A. Wright, and M. Shapshak, The double oval UV auroral distribution. 1. Implications for the mapping of auroral arcs, J. Geophys. Res., 100, 1995.

Feldstein, Y. I. and Y. I. Galperin, The auroral luminosity structure in the high-latitude upper atmosphere: its dynamics and relationship to the large-scale structure of the Earth's magnetosphere, Rev. Geophys. Space Phys., 23, 217-275, 1985.

Forget, B., J. -C Cerisier, A. Berthelier, J. J. Berthelier, Ionospheric closure of small-scale Birkeland currents, J. Geophys. Res. 96, 1843-1847, 1991.

Fukunishi, H., R. Fujii, S. Kokubun, F. Tohyama, T. Mukai, H., Oya (Small scale field aligned currents observed by the AKEBONO (EXOS-D) satellite, Geophys. Res. Lett., 18, 297, 1991.

Galperin, Y. I., Prebreakup arc morphology and the cross-tail line current model, SUBSTORMS 1, Ed. C. Mattok, ESA SP-335, Noordwijk, 263-271, 1992
Galperin, Y. I., Stable auroral arcs - observations and models, SUBSTORMS 2, Proceedings of the Second International Conference on Substorms, Fairbanks, Alaska, USA; Eds J. R. Kan, J. Craven, and S. -I. Akasofu, University of Alaska Fairbanks, 1994.

Galperin, Y. I., Magnetospheric tail structure: concepts, problems, and storm-time development of the auroral oval, J. Atmos. Terr. Phys., 57, 1397-1414, 1995.

Galperin, Y. I. and Y. I. Feldstein, Auroral luminosity and its relationship to magnetospheric plasma domains, in Auroral Physics, Eds. C.-I. Meng, M. J. Rycroft and L. A. Frank, Cambridge UP, Cambridge. 207-222, 1991.

Galperin, Y. I. and Y. I. Feldstein, Mapping of the precipitation regions to the plasma sheet, J. Geomag. Geoelectr., (in press), 1996.

Galperin, Y. I., Y. N. Ponomarev, and V. M. Sinitsin, Some algorithms for calculations of reference geophysical information along orbits of near-Earth satellites, Preprint IKI, 554, 1-42, 1980

Galperin, Y. I., A. V. Volosevich, and L. M. Zelenyi, Pressure gradient structures in the tail neutral sheet as "Roots of the Arcs" with some effects of stochasticity, Geophys. Res. Lett., 19, 2163-2166, 1992

Girard, L., and C. Senior, Combined EISCAT and all-sky camera observations of auroral structures, Geophys. Res. Lett., 18, 1185-1188, 1991.

Horwitz, J. L., Doupnik, J. R. Banks, P. M. Chatanika radar observations of the latitudinal distributions of auroral zone electric fields, conductivities, and currents, J. Geophys. Res., 83 1463, 1978.

Kan, J. R., Towards a unified theory of discrete auroras, Space Sci. Rev., 31, 71-117, 1982.

Kan, J. R., L. Zhu, and S. -I. Akasofu, A theory of substorm: onset and subsidence, J. Geophys. Res., 93, 5624-5640, 1988

Kotikov, A. L., A. V. Frank-Kamenetsky, Yu. O. Latov, O. A Troshichev, E. M. Shishkina, J. S. Murphree, and R. D. Elphinstone, Filamentary structure of westward electrojet, $J$. Atmos. Terr. Phys., 55, 1763-1774, 1993.

Lanchester, B. S., and D. D. Wallis, Magnetic field disturbances over auroral arcs observed from Spitsbergen, J. Geophys. Res., 90, 2473-2480, 1985

Liu, W. W., B. -L. Xu, J. C. Samson, and G. Rostoker, Theory and observations of auroral substorms: a magnetohydrodynamic approach, J. Geophys. Res., 100, 79-95, 1995.

Lu, G., Reiff. P. H., Burch, J. L. and Winningham J. D, On the auroral current-voltage relationship, J. Geophys. Res., 96, 3523, 1991.

Luhr, H., J. Warnecke, L. J. Zanetti, P. -A. Lindkvist, and T. J. Hughes, Fine structure of field-aligned current sheets deduced from spacecraft and ground-based observations initial FREJA results, Geophys. Res. Lett., 21, 1883-1886, 1994.

Lyons, L. R., Formation of auroral arcs via magnetosphereionosphere coupling, Rev. Geophys., 30, 93-112, 1992

Lyons, L. R., and J. C. Samson, Formation of the stable auroral arc that intensifies at substorm onset, Geophys. Res. Lett., 19, 2171-2174, 1992.

Lysak, R. L., Electrodynamic coupling of the magnetosphere and ionosphere, Space Sci. Rev., 52, 33, 1990.

Lysak, R. L., Feedback instability of the ionospheric resonant cavity. J. Geophys. Res., 96, 1553-1568, 1991

Maggs, J. E., and T. N. Davis, Measurements of the thicknesses of auroral structures, Planet. Space Sci., 16, 205, 1968

Nadubovich, Y. A., and G. V. Starkov, Fibrous structure of weak homogeneous auroral arcs, Geomagn. Aeron. (in Russian), 2, 71-73, 1962.

Newell, P. T., S. Wing, C. -I. Meng, and S. Sigillito, The auroral oval position, structure and intensity of precipitation from 1984 onwards: an automated online data base, J. Geophys. Res., 96, 5877-5882, 1991.

Obara, T., T. Mukai, H. Hayakawa, A. Nishida, K. Tsuruda, S., Machida and H. Fukunishi, Akebono (EXOS-D) observations of 
small-scale electromagnetic signatures relating to polar cap precipitation, J. Geophys. Res., 98, 11153-11159, 1993.

Ohtani, S., L. J. Zanetti, T. A. Potemra, K. B. Baker, J. M. Ruohoniemi and A. T. Y. Lui, Periodic longitudinal structure of field-aligned currents in the dawn sector: Large-scale meandering of an auroral electrojet, Geophys. Res. Lett., 21, 18791882, 1994.

Oguti T., TV observations of auroral arcs, in Physics of auroral arc formation, Eds. S. -I. Akasofu, and J. R. Kan, AGU, Geophys Monogr. 25, Washington, 31-41, 1981.

Opgenoorth, H. J., M. A. L. Persson, T. I. Pulkkinen, and R. J. Pellinen, Recovery phase of magnetospheric and its association with morning-sector aurora, J. Geophys. Res., 99, 4115-4129, 1994.

Potemra, T. A., M. J. Engebretson, L. J. Zanetti, R. E. Erlandson, P. F. Bythrow, Satellite observations of currents and waves in space plasmas, laser and particle Beams, Vol. 6, Part 3, 503, 1987.

Pulkkinen, T. I., D. N. Baker, C. J. Owen, J. T. Gosling, and N. Murphy, Thin current sheets in the deep geomagnetic tail. Geophys. Res. Lett., 20, 2427-2430, 1993.

Pulkkinen, T. I., D. M. Baker, R. J. Pellinen, J. S. Murphree, and L. A. Frank, Mapping of the auroral oval and individual arcs during substorms, J. Geophys. Res., 100, 21987-21994, 1995.

Rankin, R., P. Frycz, V. T. Tikhonchuk, and J. C. Samson, Nonlinear standing shear Alfven waves in the Earth's magnetosphere, J. Geophys. Res., 99, 21291-21301, 1994.

Rothwell, P. L., M. B. Silevitch, L. P. Block, and C. -C. Falthammar, Prebreakup arcs: a comparison between theory and experiment, J. Geophys. Res., 96, 13967-13975, 1991.

Samson, J. C., and R. Rankin, The coupling solar wind energy to MHD cavity modes and field line resonances in the Earth's magnetosphere, in Solar Wind Sources of Magnetospheric ULF Waves Eds. M. J. Engebretson, K. Takahashi, and M. Scholer, Geophys. Monograph Ser., Vol 81, Washington, D.C., 253, 1994.

Samson, J. C., D. D. Wallis, T. J. Hughes, F. Creutzberg, J. M. Ruohoniemi, and R. A. Greenwald, Substorm intensifications and field line resonances in the magnetosphere, J. Geophys. Res., 97 8495-8518, 1992.

Sato, T., A theory of quiet auroral arcs. J. Geophys. Res., 83, 1042-1048, 1978.

Sesiano, J., and P. A. Cloutier, Measurements of field-aligned currents in a multiple auroral arc system, J. Geophys. Res., 81, 116-122, 1976.

Stormer, C., The polar aurora, Clarendon Press, Oxford, 1955.

Timofeev, E. E. and Y. I. Galperin, Convection and current in stable auroral arcs and inverted-V's, J. Geomag. Geoelectr., 43, Suppl., 259-274, 1991.
Trakhtengertz, V. Y. and A. Y. Feldstein, Quiet auroral arcs: ionosphere effect of magnetospheric convection stratification, Planet Space Sci., 32, 127, 1984.

Trakhtengertz, V. Y., and A. Y. Feldstein, Turbulent regime of magnetospheric convection, Geomagn. Aeron. (in Russian), 27, 258-264, 1987a.

Trakhtengertz, V. Y., and A. Y. Feldstein, Excitation of small-scale electromagnetic disturbances in the Ionospheric Alfven Resonator, Geomagn. Aeron. (in Russian), 27, 315-317, $1987 \mathrm{~b}$.

Trakhtengertz, V. Y., and A. Y. Feldstein, Turbulent Alfven boundary layer in the polar ionosphere. 1. Excitation conditions and energetics. J. Geophys. Res., 96, 19363-19374, 1991.

Traver, D. P., D. G. Mitchell, and D. J. Williams, Statistical study of the late substorm recovery phase and quiet time plasma sheet based on ISEE $1 \sim 30-\mathrm{keV}$ ion observations, J. Geophys. Res., 99, 10981-10994, 1994.

Triska, P., F. Jiricek, V. Velicky, and J. Vojta, A subsatellite for mother-daughter active space experiments, Adv. Space Res., 10, (7), 165, 1990.

Tverskoy, B. A., Nature of homogeneous polar auroral arcs, Geomagn. Aeron., 22, 794-799, 1982 a.

Tverskoy, B. A., Longitudinal currents in the magnetosphere, Geomagn. Aeron. 22, 812-815, 1982b.

Velichko, V. A., S. I. Soloviev, N. E. Molochushkin, and V. P. Samsonov, Brightening of an auroral arc and its structure variation during a strong substorm, Geomagn. Aeron. (in Russian), 27, 850-852, 1987.

Volokitin, A. S., V. V. Krasnoselskikh, E. V. Mishin et al., On small-scale structure of intense field-aligned currents at high latitudes, Cosmic Res. (in Russian), 22, 749-755, 1984.

Watanabe, T., H Oya, K. Watanabe, and T. Sato, Comprehensive simulation study on local and global development of auroral arcs and field-aligned potentials, J. Geophys. Res., 98, 2139121407, 1993

Yamamoto, T., E.Kaneda, H. Hayakawa, T. Mukai, A. Matsuoka, S. Machida, H. Fukunishi, N. Kaya, K. Tsuruda and A. Nishida, Meridional structures of electric potentials relevant to premidnight discrete auroras: a case study from Akebono measurements, J. Geophys. Res., 98, 11135-11151, 1993.

Zhu, L., J. J. Soika, R. W. Schunk, and D. J. Crain, A timedependent model of polar cap arcs, J. Geophys. Res., 98, 61396150, 1993.

Zhu, L., J. J. Soika, R. W. Schunk, and D. J. Crain, Model study of multiple polar cap arcs: occurrence and spacing, Geophys. Res. Lett., 21, 649-652, 1994. 